\title{
Fallbericht: Elektiver Mutismus
}

\section{"Ich habe große Angst, etwas falsch zu machen..."}

\author{
Eine 19-jährige Studentin hat ihr mündliches Abitur in Biologie mit \\ 12 Punkten bestanden - ohne ein Wort zu sagen. Jetzt benötigt sie \\ ihre Stimme, um soziale Kontakte aufzubauen und ihr Studium \\ erfolgreich zu beenden.
}

\section{Erstvorstellung}

Mündliche Kommunikationsschwierigkeiten Frau P. (18 Jahre) kommt auf eigenen Wunsch zur ambulanten Psychotherapie. Sie kann sich nur schwer mündlich mitteilen und berichtet größtenteils schriftlich, dass sie Schwierigkeiten habe, mit ihr nicht vertrauten Personen zu sprechen.

Beginn der Symptomatik Dies habe in der 7. Klasse im Gymnasium begonnen. Damals habe die Geschichtslehrerin sie in der Pause gefragt, weshalb sie nicht zu ihren Mitschülern ginge. Anschließend habe sie zunächst im Geschichtsunterricht nicht mehr gesprochen und dies mit der Zeit auf alle Fächer ausgeweitet. Die Pause habe sie meist mit 2 Freundinnen verbracht. Mit diesen sei sie Runden im Schulhof gelaufen. Sie sei aber nicht immer gefragt worden, ob sie mitlaufen wolle, und sei nicht immer ins Gespräch einbezogen worden.

Schüchternheit von jeher In der Grundschulzeit habe sie noch gesprochen. Ihren Lehrern sei damals aber aufgefallen, dass sie sich nicht melde und nur auf Ansprache antworte. Sie hätten daraufhin ihre Eltern darauf angesprochen, dass ihre Tochter sehr schüchtern und still sei. Die Eltern fanden das Verhalten ihrer Tochter jedoch normal.

Erstarren bei sozialem Kontakt Sie erlebe soziale Situationen als unangenehm. In diesen Situationen falle sie meist in eine
Art Starre und könne weder sprechen noch sich bewegen. Sie sei grundsätzlich sehr schreckhaft und unsicher und versuche Kontakte zu Menschen und Gespräche zu vermeiden, ebenso Referate im Rahmen ihres Studiums.

Scham-Angst Sie denke, andere würden sie nicht beachten, sich nicht für sie interessieren. Gerade im Gymnasium habe sie häufig erlebt, dass sich Mitschüler nicht für sie interessierten und sie nicht beachteten. Hinzu komme eine große Angst, etwas falsch zu machen. Sie befürchte, eine falsche Frage zu stellen oder etwas Unpassendes zu sagen.

Entscheidungsschwierigkeiten Bei alltäglichen Dingen könne sie sich oft nicht entscheiden, da sie immerzu abwäge. Wenn ich ihr im Therapieraum die Entscheidung überlasse, auf welchen Stuhl sie sich setzen möchte, „erstarrt“ sie und kann keine Bewegung mehr umsetzen, bis ich die Entscheidung übernehme. Ebenso fällt es ihr sehr schwer, zuerst durch die Tür in den Flur zu gehen. Sie stockt dann und benötigt viel Zeit, um die Schritte aus dem Therapieraum hinaus zu wagen.

Beeinträchtigung im Alltag In Alltagssituationen, etwa beim Einkaufen oder im Restaurant, könne Frau P. meist keine Antworten geben oder spreche dann so leise, dass ihr Gegenüber sie nicht verstehen könne. Dies beeinträchtige sie in ihrem Alltag sehr und bringe sie immer in erhöhte psychophysiologische Anspannung.

\section{Beobachtete Symptome}

Zwangssymptome Im Kontakt fällt auf, dass Frau P. alles ordnet und immer die gleichen Handlungen vollführt. Z.B. ordnet sie ihre Hefte und Blöcke im Rucksack in eine bestimmte Reihenfolge und zieht ihre Jacke und ihren Schal auch immer in gleicher Art und Weise an. Dieses Verhalten beeinträchtigt sie nicht.

Verkrampfung bei Unerwarteten Ereignissen Etwas Unerwartetes bringt sie in eine deutliche, von außen sichtbare Anspannung. In dieser Anspannung verkrampft sich ihr Körper, und sie verletzt sich selbst in Form von Beißen, Kratzen und Kopf gegen die Wand schlagen.

Schreckhaftigkeit Außerdem besteht eine sehr stark ausgeprägte Schreckhaftigkeit, die bei Türknallen oder Geräuschen auBerhalb des Therapieraums zu Zuckungen bei der Patientin führt.

\section{Vorbehandlung}

Frau P. sei 2008/2009 über 9 Monate hinweg in ambulanter psychotherapeutischer Behandlung gewesen. Ihr Symptomatik sei nur wenig besser geworden, und die Bezie- 
hung zum Therapeuten sei nicht so gut gewesen. Deshalb habe sie abgebrochen.

\section{Biografische Anamnese}

Familienkonstellation Frau P. sei bei ihren Eltern als jüngstes von 4 Kindern gemeinsam mit 3 Schwestern $(+7,+4,+2$ Jahre) aufgewachsen. Sie sei sehr behütet großgeworden und habe ein gutes Verhältnis zu ihrer Familie. Besondere Vorkommnisse oder traumatische Ereignisse könne sie keine benennen.

Verhältnis zu den Geschwistern Zu ihren Schwestern habe sie ein geteiltes Verhältnis: Einerseits verstünden sie sich gut, mit einer Schwester habe sie früher aber viele Konflikte gehabt und diese Schwester als rücksichtslos erlebt. Mit einer anderen Schwester fühle sie sich im Kontakt teils unwohl, da sie häufig das Gefühl habe, etwas falsch zu machen und die Schwester zu verletzen. Bei fremdanamnestischen Gesprächen mit der Schwester fiel auf, dass diese der Patientin ggü. grenzüberschreitend ist.

Verhätlnis zur Mutter Als sie 10 Jahre alt war, habe ihre Mutter unter psychosomatischen Schmerzen gelitten und sich eine Zeit lang häufig mit einer der Schwestern gestritten. Anschließend habe ihre Mutter sie gefragt, ob sie keine gute Mutter sei und ob sie nicht Recht habe. Die Patientin habe ihre Mutter in solchen Momenten getröstet. Zusätzlich sei sie sehr bemüht gewesen, keine Last zu sein, und habe ihre eigenen Schwierigkeiten in der Schule der Mutter nicht zumuten wollen.

Aktuelle familiäre Situation Die Patientin fahre noch immer regelmäßig an den Wochenenden zu ihren Eltern. Es falle ihr schwer, sich ggü. der Familie abzugrenzen und Nein zu sagen, insbesondere ggü. der Mutter. Mit ihrer Familie und ihr sehr vertrauten Personen spricht die Patientin.

Schulischer Werdegang Sie habe die Grundschule besucht, ihr Abitur mit der
Note 2,2 gemacht (mündliche Prüfung in Biologie ohne zu sprechen) und studiere nun Erziehungswissenschaften. Ihre Noten litten jedoch unter ihren Problemen, und auch der beeinträchtigte soziale Kontakt mit anderen Menschen belaste sie.

Kontakt zu Gleichaltrigen Derzeit lebe sie zusammen mit einer Kommilitonin in einer Wohngemeinschaft. Mit dieser könne sie sprechen. Mit anderen Kommilitonen an der Universität spreche sie i.d.R. nicht, außer sie habe eine Frage, bei der sie sicher sei, dass diese gerechtfertigt ist.

Freizeitaktivitäten Seit ihrer Kindheit sei sie in einer Tanzgruppe. Dies falle ihr hin und wieder schwer, aber sie mache dort mit und könne in der Gruppe auch vortanzen.

Partnerschaft Bislang sei sie noch keine partnerschaftliche Beziehung eingegangen, habe aber Interesse daran.

\section{Psychischer Befund}

- Altersentsprechend wirkende Patientin, bewusstseinsklar, allseits orientiert.

- Aufmerksamkeit, Konzentration und Gedächtnis scheinen ungestört.

- Formales Denken geordnet, inhaltliches Denken gekennzeichnet durch Selbstzweifel und Gedanken an Minderwertigkeit, Unsicherheit.

- Wahrnehmungsstörungen liegen nicht vor.

- Teils dissoziative Zustände erkennbar.

- Starr, mutistisch.

- Intermittierende Anspannungszustände bei Fragen.

- Kein Blickkontakt.

- Grimassiert, monotone Mimik wechselnd mit dramatischen Zügen.

- Auf Fragen ansprechbar, scheint erreichbar.

- Auffallend geräuschempfindlich.

- Im Affekt ängstlich, gedrückt, teils Wut spürbar bei erhaltener Schwingungsfähigkeit.

- Antrieb unauffällig.
- Psychomotorik angespannt, gehemmt.

- Lebensüberdrussgedanken.

- Keine akute Suizidalität.

\section{Testbefunde}

- In der SCL-90 (Franke, 2002) zeigen sich erhöhte Werte auf den Skalen Ängstlichkeit (62) und Somatisierung (60).

- Im Persönlichkeitsstil und -störungs Inventar (PSSI, Kuhl \& Kazén, 2009) zeigen folgende Skalen erhöhte Werte:

$\triangleright$ schizoid (61),

$\triangleright$ selbstunsicher (69),

$\triangleright$ zwanghaft (76),

$\triangleright$ abhängig (66),

$\triangleright$ borderline (60),

$\triangleright$ depressiv (69).

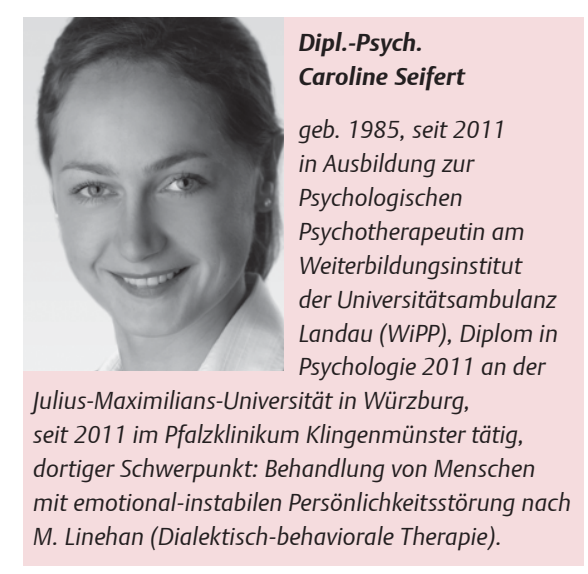

mit emotional-instabilen Persönlichkeitsstörung nach

M. Linehan (Dialektisch-behaviorale Therapie). 


\section{Kommentare zum Fallbericht}

\section{Analytische Perspektive}

Frühes Trauma Da sich die Patientin an kein Trauma erinnert, scheint es vor der Sprachentwicklung zu liegen. Die Patientin erhielt damals vermutlich wenig vitalisierende Zuwendung durch die Eltern und der Blickkontakt war gestört. Evtl. litt die Mutter bereits unter Erschöpfung, da spätere psychosomatische Störungen erwähnt werden.

Rollentausch Die Patientin ist eindeutig parentifiziert, wie durch ihre Sorge um die Mutter deutlich wird. Diese scheint erhebliche Zweifel an ihrer mütterlichen Qualität zu haben, da sie diesbezüglich bei ihrer eigenen Tochter um Rückversicherung fragt. Die Patientin muss die Mutter trösten; sie mutet ihr keine Schwierigkeiten zu, hat keinen Rückhalt. Evtl. hatten die Eltern sogar einen Jungen erhofft und das weibliche Selbstwertgefühl der Patientin ist deshalb schwach. Bezeichnenderweise verstärkt sich das Symptom ab der Pubertät.

Geschwisterkonflikte Eine Schwester ist, vermutlich aus früher Rivalität ggü. dem Nesthäkchen, übergriffig und grenzüberschreitend (Erschrecken, Kneifen, Schlagen, Anschreien, Ablehnenung?) - dies könnte eine frühe Schreckstarre und Angst hervorgerufen haben. Die Angst der Patientin, etwas Unpassendes zu sagen, könnte auf Gelächter und Hänseleien der Geschwister zurückzuführen sein. Ein böses Introjekt der Selbstablehnung, möglicherweise sogar ein frühes Abschalten unerträglicher Gefühle führt zur Erstarrung. Dass die Eltern das Verhalten „normal“ finden, weist auf extrem mangelnde Einfühlung und Sensibilität hin, es besteht der Verdacht auf Narzissmus. Offenbar gab es häufig Konflikte mit den älteren Schwestern und es herrschte eine unruhige, laute, ängstigende Atmosphäre.

Beschützende Atmosphäre anbieten Ich würde die Patientin nicht unter Druck setzen zu sprechen, sondern sie zum Liegen bitten, mich daneben setzen, ihren Atem beachten, geduldig warten, eine haltende Hand und eine beschützende Atmosphäre anbieten. Anstatt eine als bedrohlich und fordernd erlebte Beziehung würde ich mich im Sinne von Winnicott oder Balint als „bergende Substanz“ anbieten, nicht als konturiertes Objekt (Uterusatmosphäre), weiterhin vorsichtig Blickkontakt mit Einfühlung statt Deutung anbieten - offensichtlich hat die Patientin Angst vor „Blick“, vor Beziehung.

Arbeit mit Rollenspielen Nach seelischer Kräftigung der Patientin würde ich mit Rollenspielen arbeiten: Wichtige Personen (notfalls Introjekte) werden auf einen leeren Stuhl gesetzt und die Patientin ermutigt, sie zu benennen und zu ihnen über vorherrschende gute und negative, bisher unausgesprochene Gefühle zu sprechen. Dabei ist oft Ermutigung notwendig, sogar ein Vorsprechen durch den Therapeuten als fühlenden Stellvertreter. Wenn dies gelingt, kann ein Rollenwechsel vorgeschlagen werden: Die Patientin identifiziert sich mit der angesprochenen Person, setzt sich auf deren Platz und versucht, sich einzufühlen. Danach kann man zu einfühlsamen Deutungen übergehen und die bisherige Lebensleistung trotz der beschämenden Behinderung und Einschränkung würdigen.

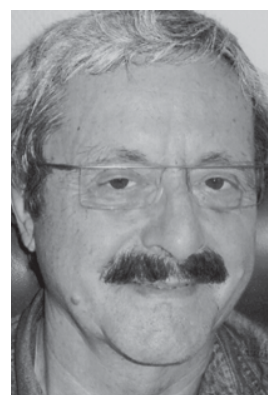

Tilmann Moser

geb. 1938, Studium der Literaturwissenschaft in Tübingen/Berlin/Paris, journalistische Ausbildung in Stuttgart, danach Studium der Soziologie, Promotion über Jugendkriminalität, Ausbildung zum Psychoanalytiker am Sigmund-Freud-Institut in Frankfurt, 1969-1978 Dozent dort am Fachbereich Jura, seit 1978 private Praxis in Freiburg; Schwerpunkte: Psychoanalyse und Spätfolgen des Dritten Reiches, Psychoanalyse und Körperpsychotherapie, Psychoanalyse und religiöse Störungen.

\section{Verhaltenstherapeutische Herangehensweise}

Ressourcen Zunächst wirkt alles eng und wenig beweglich: Ist nicht das Beispiel der mündlichen Abiturprüfung geeignet, dem Therapeuten die Verfestigung von Mustern vor Augen zu führen? Auf den 2. Blick entdecke ich eine ganze Reihe von Ressourcen: Nach längerer Pause begibt sich diese junge Frau erneut in Behandlung. Was ist ihre Motivation für den erneuten Anlauf? Welcher Wunsch verbirgt sich hinter der Wahl des Studienfachs Erziehungswissenschaften? Wie schafft sie es, die Schwelle beim Vortanzen zu überwinden? Was verbindet sie mit dem Wunsch nach einer Partnerschaft?

Auftragsklärung Im Rahmen der Auftragsklärung würde ich auch überprüfen, ob es ihr mehr um den Aspekt des freien Sprechens geht, um mehr Sicherheit in sozialen Situationen oder um das Erreichen eines Zustands, in dem sich das Zusammensein mit anderen Menschen gut anfühlt. Wäre sie auch mit teilweiser Zielerreichung zufrieden? Diese Herausarbeitung kann auch zur Konturierung von bisher noch sehr diffusen Zielen führen.

Weitere Aspekte Welche Signale der Sicherheit, wieviel Klarheit im therapeutischen Kontrakt kann ich ihr anbieten? Spielen Gender-Aspekte eine Rolle? Wie ist die Lerngeschichte der Patientin? Kann man aus dem Gelingen der verbalen Kommunikation im familiären Umfeld und in der Wohngemeinschaft etwas auf den therapeutischen Kontext übertragen? Kann ich mit ihr ein gemeinsames Konzept zur bisherigen Aufrechterhaltung der „Störung“ entwickeln? Welche Lösungen hat sie in der Vergangenheit erfolgreich angewandt? Was kennzeichnet die Situationen, in denen Sprechen kein Problem ist oder leichter fällt? Ist Singen, etwa in einem Chor, eine nützliche und nutzbare Facette des Stimmgebrauchs? Welche 
Ressourcen sind mit dem Tanzen verbunden? Was entsteht aus der Beweglichkeit, die sie dort entwickelt? Bietet Erstarrung in anderen Situationen Schutz, oder ist sie Ausdruck empfundener Ohnmacht?

Zur Familie Der Vater ist nicht erwähnt, die Einengung in Rollen und unangemessene Rollenübernahmen werden beschrieben. Gleichzeitig gibt es Hinweise auf Geborgenheit. Dies wären meine Ansatzpunkte, um Ideen zu generieren für die Gestaltung der menschlichen Beziehungen, die auch in der Zukunft wichtig sein werden.

Hilfsmittel An einem Seil als „Lebensfaden“ entlang ließen sich z.B. kleine Schlüsselszenen des Gelingens oder Scheiterns anheften. Schreiben, Skizzieren, großflächiges Illustrieren auf groß dimensionierten Papierbögen könnte mich als Therapeuten und evtl. auch die Patientin von dem anfänglichen Gefühl der Enge befreien. Es könnte helfen, den bereits angelegten Wunsch der Erweiterung eigener Möglichkeiten lebendiger werden zu lassen.

Verhaltenstherapeutische Arbeit Erst dann wird wohl der Blick frei auf ein sinnvolles Einüben von Kommunikation, nach eigenen Prioritäten und außerhalb des Therapiezimmers. Das Arbeiten am Defizit wäre mir dabei genauso wichtig wie die Entdeckung genussvollen Erlebens, das gezielte Aufsuchen angenehmer Situationen, in denen Selbstfürsorge möglich wird.

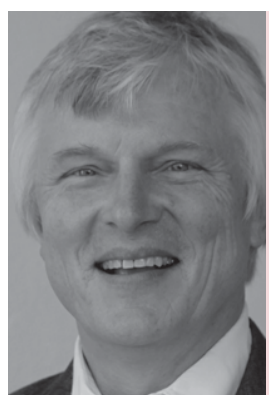

\section{Dr. med. Michael Brünger}

geb. 1954, Arzt für Kinder- und Jugendpsychiatrie und -psychotherapie, Verhaltenstherapeutischer Lehrtherapeut und Supervisor, systemischer Familientherapeut, Psychotraumatherapeut. Chefarzt im Pfalzinstitut - Klinik für Kinder- und Jugendpsychiatrie, Psychosomatik undPsychotherapie in Klingenmünster/Rheinland-Pfalz.

\section{Systemisch-integrativer Blickwinkel}

Eingangsfragen Aus systemisch-integrativer Perspektive interessiert mich zunächst die junge Frau als Mensch: Wie nimmt sie Kontakt auf? Was macht sie gerne? Welche Interessen, Vorlieben, Abneigungen hat sie? Wie organisiert sie ihren Alltag? Wie sieht ihr Ressourcenprofil aus? Um das Abitur gemacht und ein Studium aufgenommen zu haben, muss sie über erhebliche soziale Kompetenzen verfügen, um andere Personen zu aktivieren, auf ihre „Schwäche“ Rücksicht zu nehmen und ihr zu helfen und entgegenzukommen. Mithilfe einer Beziehungslandkarte würde ich mir zeigen lassen, in welchen Personen-, Orts- und Zeitkontexten ihr das Sprechen leich- ter bzw. schwerer fällt. Manchmal verschwindet die Symptomatik nach einem Umzug oder beim Sprechen einer Fremdsprache.

Familienarbeit Aus systemischer Sicht wäre es nahliegend, die Familie - zumindest für einige Gespräche - stärker einzubeziehen und dabei u.a. folgenden Fragen nachzugehen: Wie wird mit dem Fremden, wie mit dem Vertrauten umgegangen? Wer kann sich wohlfühlen in der Fremde, wer hat das Vertrauen in sich, gut zurecht zu kommen, wo auch immer es sei? Erwähnt wird eine Hyperakusis: Ich würde die Entwicklungsgeschichte der Patientin erfragen und nach Teilleistungsschwächen und möglichen Sprachentwicklungsproblemen forschen. Wie kommunizieren die Eltern miteinander? Gibt es einen lustvollen Umgang mit Sprache, lohnt es sich, etwas zu sagen, oder besteht das Gefühl, ohnehin kein Gehör zu finden? Ähnlich wie bei Magersucht verleiht die Weigerung zu sprechen durch die Macht des Neins eine gewisse Stärke.

Alternative Kommunikationsformen Es ist nicht möglich, nicht zu kommunizieren. Was würde sich ändern, wenn die Patientin sich über ein Laptop mit Sprachausgabe mitteilte? Sie würde nicht sprechen und dennoch sprechen (lassen). Anstatt sich in einen Machtkampf hinziehen zu lassen, ob gesprochen wird oder nicht, würde ich prüfen, welche nichtsprachlichen Kommunikationsformen (z. B. Spielen, Zeichnen, Singen, Bewegung, Gesten, Sprechen mit den Augen, Sprechen von Nonsense-Silben, unhörbar leise sprechen, etc.) andere Möglichkeiten des Austauschs eröffnen.

Perspektive Über die Vorbehandlung ist wenig bekannt. Was hatte ihr dort nicht zugesagt? Wenn sie tatsächlich so unsicher ist, wie war es ihr möglich, die Behandlung zu beenden? Was müsste passieren, damit die gegenwärtige Therapie gut läuft, was würde ihr umgekehrt nicht gut tun? Entscheidend für den weiteren Behandlungsverlauf dürfte es sein, ob die Patientin sich für Veränderungsschritte entscheidet oder sich zu sehr an ihr Symptom und die geschaffenen besonderen Beziehungsgefüge gewöhnt hat.

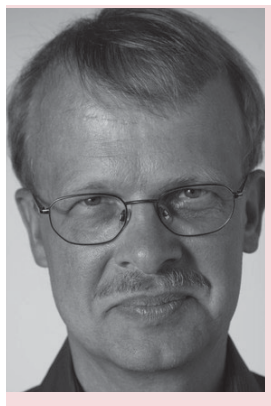

Dr. sc. hum. Rüdiger Retzlaff, Dipl.-Psych.

geb. 1957, Psychotherapeut, Kinder- und Jugendtherapeut, Lehrtherapeut für systemische Therapie und systemische Therapie mit Kindern und Jugendlichen, Supervisor für Hypnotherapie, psychodynamische Therapie und Verhaltenstherapie, Lehrtherapeut am Helm Stierlin Institut Heidelberg, Leiter der Ambulanz für Paar- und Familientherapie am Institut für Psychosomatische Kooperationsforschung und Familientherapie, Zentrum für Psychosoziale Medizin der Universitätsklinik Heidelberg; niedergelassen in eigener Praxis in Heidelberg.

Beitrag online zu finden unter

http://dx.doi.org/10.1055/s-0034-1370827 